\title{
ORIGINAL
}

\section{LA PERSPECTIVA DE LAS CUIDADORAS INFORMALES SOBRE LA ATENCIÓN DOMICILIARIA. UN ESTUDIO CUALITATIVO CON AYUDA DE UN PROGRAMA INFORMÁTICO (*)}

\author{
M. ${ }^{a}$ Ángeles Prieto Rodríguez (1), Eugenia Gil García (2), Cristina Heierle Valero (3) y Antonio \\ Frías Osuna (4). \\ (1) Escuela Andaluza de Salud Pública, Granada \\ (2) Hospital Universitario San Cecilio, Granada. \\ (3) Escuela Universitaria de Enfermería «Virgen de las Nieves», Granada. \\ (4) Universidad de Jaén. Departamento de Ciencias de la Salud. \\ (*) Este estudio ha sido financiado por el FIS: 98/0653 y por la Consejería de Salud de la Junta de Andalucía: 23/97.
}

\section{RESUMEN}

Fundamento. Existe en nuestro país un vivo debate sobre los modelos de atención domiciliaria que se deben desarrollar. Este estudio pretende conocer cómo perciben la calidad de la atención domiciliaria los cuidadores familiares de pacientes con cáncer en fase terminal, ancianos con demencia y personas intervenidas en programadas de cirugía mayor ambulatoria.

Métodos: Estudio cualitativo de tipo fenomenológico basado en grupos de discusión (9), grupos triangulares (5) y entrevistas en profundidad (22). El estudio se realizó en Andalucía, durante los años 1999 y 2000. Los sujetos del estudio son los cuidadores principales de los pacientes que reciben atención domiciliaria desde los centros de salud. La información se ha analizado mediante análisis de contenido apoyado por el software NUDIST- 4. Las variables de análisis han sido las del modelo SERVQUAL.

Resultados. Para las cuidadoras de pacientes con cáncer las dimensiones más importantes de la calidad de la atención domiciliaria son la Capacidad de Respuesta y la Accesibilidad. Elementos negativos que perjudican la accesibilidad son: la falta de cobertura de atención domiciliaria fuera del horario normal, la dificultad de contactar por teléfono, la tardanza en acudir y las visitas sólo a demanda. Las cuidadoras de pacientes intervenidos en cirugía mayor ambulatoria quieren Seguridad y la Fiabilidad. Las cuidadoras de ancianos con demencia consideran prioritario que se les proporcionen los materiales que necesitan para cuidar al enfermo.

Conclusiones: Las expectativas de cuidadores y pacientes varían en función del problema de salud. Por lo tanto, las características de la atención domiciliaria tendrán que variar también en función de ello. Se hace necesario un modelo flexible que sea capaz de adaptarse a las necesidades de los diferentes tipos de pacientes y las circunstancias también distintas de sus cuidadores familiares.

Palabras clave: Atención domiciliaria. Satisfacción de usuarios. Calidad percibida. Paciente terminal. Ancianos dependientes. Cirugía ambulatoria. Métodos cualitativos.

\section{Correspondencia:}

M. ${ }^{a}$ Ángeles Prieto Rodríguez

Escuela Andaluza de Salud Pública

Campus universitario de Cartuja.

Apartado de correos 2070 Granada 18080

Correo electrónico: prieto@easp.es

\section{ABSTRACT}

The Perspective of Non-Institutional Caregivers Regarding Home Care in Andalussia. A Computer-Aided Qualitative Study

Background: A hot debate exists in our country as to the models of home care which must be developed. This study is aimed at ascertaining how the family caregivers of terminal cancer patients, of the elderly suffering from dementia and of individuals having undergone major operations in outpatient surgery programs rate the quality of the home care provided.

Methods: A phenomenological type qualitative study based on discussion groups (9), triangular groups (5) and in-depth interviews (22). This study was conducted in Andalusia throughout the 1999-2000 period. The subjects of the study were the main caregivers of patients provided with home care through the healthcare centers. The information must be analyzed by means of a Nudist-4 software-aided content analysis. The analysis variables were those of the Servqual model.

Results: For the caregivers of cancer patients, the most important aspects of the quality of the home care provided were the Response Capacity and Accessibility. This analysis revealed that the patients suffered pain but the pain was not controlled. Negative aspects hindering accessibility were the lack of home care coverage outside of regular working hours, the difficulty of getting in touch by phone, the length of time it takes for someone to come and the visits solely on request. The caregivers of patients having undergone major outpatient surgery want Security and Reliability. They complain of the short length of time within which the patients are released from the hospital and of the home care provided by the health care center. The caregivers of the elderly with dementia place top priority on being provided with the materials they need to take care of these patients.

Conclusions: Caregivers' and patients' expectations differ, depending on health problems, therefore, the type of home care provided should vary, according to the health problems involved. It is necessary to develop a flexible model, capable of adapting to different patient needs and the diverse circumstances that affect family caregivers.

Keywords: Home Nursing. Home Care Services. Patient Satisfaction. Terminally Ill. Frail Elderly. Ambulatory Surgical Procedures. Qualitative Research. 


\section{INTRODUCCIÓN}

La oferta de servicios desde la atención primaria se está modificando en un intento de dar respuesta a las necesidades y preferencias del público y a sus problemas de salud, tanto prevalentes como de nueva aparición. Y esto, bajo una premisa básica: la eficiencia como base de todas las actuaciones y la satisfacción de los ciudadanos como meta $^{1}$.

El contexto socio sanitario es el punto de partida para la orientación de los servicios. $\mathrm{Y}$ entre las variables que más le influyen hay que destacar el aumento de enfermedades crónicas invalidantes y el incremento de la esperanza de vida, que con el replanteamiento del papel de los hospitales, los cuales se están convirtiendo en hospitales «de agudos», y el desarrollo de nuevas formas familiares, en las que la mujer trabaja también fuera de casa, está provocando una crisis del cuidado de personas dependientes y enfermas, que puede agravarse en los próximos años. Todo ello plantea retos importantes a los que la atención primaria intenta dar respuesta y que están provocando la necesidad de cambios en el papel de los centros de salud y de sus profesionales ${ }^{2-5}$. De hecho, actualmente, problemas de salud que hasta ahora eran atendidos en el ámbito hospitalario, son atendidos en el domicilio desde atención primaria.
Actualmente, existe en nuestro país un vivo debate sobre los modelos y fórmulas de atención domiciliaria a desarrollar o potenciar y sobre los profesionales que deben proveer este servicio. En este contexto, la opinión de los pacientes y especialmente la de sus familiares que les cuidan en casa (cuidadores informales) es fundamental para mejorar la calidad de la atención domiciliaria, adaptando esta modalidad de atención a las necesidades, problemas y expectativas de las personas que necesitan cuidados en el hogar.

Uno de los modelos teóricos más utilizados para conocer la calidad de un servicio desde la perspectiva de los clientes es el SERVQUAL (Service Quality) de Parasuraman, Berry y Zeithman ${ }^{6-9}$. Ha sido adaptado específicamente para los servicios sanitarios y está ampliamente extendido en el sector salud. En España, numerosos estudios de satisfacción de usuarios y de calidad percibida lo utilizan como soporte teórico y como base instrumental ${ }^{10-16}$. EL SERVQUAL incluye 8 dimensiones a través de las cuales se analiza cómo percibe un usuario la calidad del servicio que recibe. Estas dimensiones son: la accesibilidad, la comunicación, la cortesía, la competencia profesional, la capacidad de respuesta, la seguridad, los elementos tangibles y la fiabilidad. En la tabla 1 se describen estas dimensiones.

Dimensiones de la calidad percibida según modelo SERVQUAL (Parasuraman)

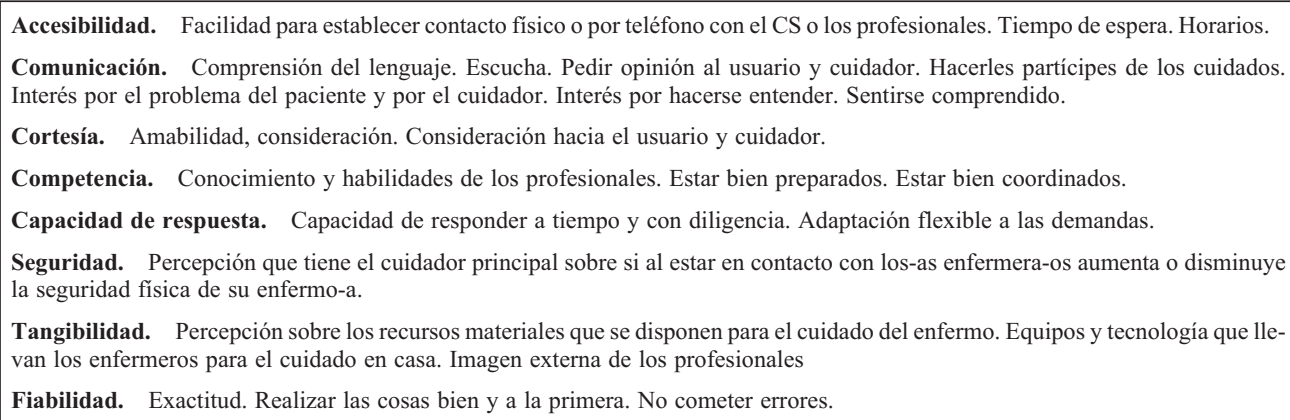

Tangibilidad. Percepción sobre los recursos materiales que se disponen para el cuidado del enfermo. Equipos y tecnología que llevan los enfermeros para el cuidado en casa. Imagen externa de los profesionales

Fiabilidad. Exactitud. Realizar las cosas bien y a la primera. No cometer errores. 
Este estudio pretende conocer cómo perciben la calidad de la atención domiciliaria (AD) proporcionada por las enfermeras de centros de salud (CS) los cuidadores familiares de pacientes con cáncer en fase terminal, ancianos con demencia y personas intervenidas en programas de cirugía mayor ambulatoria (CMA).

\section{SUJETOS Y MÉTODOS}

Tipo de estudio. Estudio cualitativo de tipo fenomenológico basado en grupos de discusión (GD), grupos triangulares (GT) y entrevistas en profundidad (E). El objetivo de este estudio: conocer cómo perciben la calidad de la AD los cuidadores familiares, explica la elección del método utilizado, que pretende entender el fenómeno de estudio desde la propia perspectiva del actor ${ }^{17}$. Las técnicas cualitativas tratan de capturar, mediante el habla del sujeto, el sentido real y multidimensional de las acciones (por qué hacen lo que hacen, por qué dicen lo que dicen) ${ }^{18-22}$.

Contexto y sujetos de estudio. El estudio se realizó en Andalucía, durante los años 1999 y 2000, entre cuidadores familiares de pacientes que eran atendidos en sus domicilios por enfermeras de centros de salud. Se han recogido opiniones de cuidadores de diferentes ciudades andaluzas, tanto en el ámbito urbano como rural. El trabajo de campo se realizó de abril a diciembre de 1999. Los sujetos del estudio son los cuidadores principales (informales) de los pacientes. Por cuidador principal definimos a la persona que asume la responsabilidad principal del cuidado en la familia ${ }^{23-25}$.

Selección de los participantes. Para la selección de los participantes se partió de una definición previa de perfiles característicos, elaborados a partir de la bibliografía revisada, de la experiencia del equipo investigador en el tema y de la información aportada por los profesionales enfermeros de los centros de salud, que actuaron como infor- mantes clave. Una vez definidos los perfiles más característicos y habituales de los cuidadores, se realizó un muestreo sistemático no probabilístico ${ }^{26,27}$, en 2 etapas:

1. Selección de la zona básica de salud, garantizando que en el estudio participaran sujetos pertenecientes al ámbito urbano y rural; 2. Selección de los cuidadores, garantizando que participaran en el estudio cuidadores de los diferentes perfiles definidos. La selección se realizó a partir de los listados de pacientes proporcionados por los enfermeros de los centros de salud seleccionados en la primera etapa, a través de un procedimiento aleatorio. Una vez seleccionado, se comprobaba en la historia y a través de una entrevista con el personal del centro, si se ajustaba al perfil y si no era así, se reemplazaba por otro mediante el mismo procedimiento. (Criterios de homogeneidad y heterogeneidad en la tabla 2). A partir de estos criterios se ha diseñado una muestra estructural. Se ha buscado el discurso de personas que representen el de su grupo de referencia y que nos permitan conocer, analizar e interpretar diferentes perspectivas. Cada perfil de sujetos incluye a su vez distintos «tipos» sociales que representan una variante discursiva ${ }^{28-31}$ (figura 1).

Técnicas de recogida de la información. En total se han realizado 3 GD, 2 GT y 10 E con cuidadores de ancianos con demencia, 3 GD y 12 E con cuidadores de pacientes en CMA y 3 GD y 3 GT con cuidadores de pacientes oncológicos terminales. La elección de una técnica $u$ otra ha estado motivada por las dificultades para formar un grupo suficiente, en número de personas, como para desarrollar una discusión grupal (como mínimo 4 personas). Esto ha ocurrido en el medio rural, donde los casos de cuidadores no permitieron reunir un número suficiente y se optó por realizar grupos triangulares o entrevistas en profundidad. También ocurrió con el perfil de cuidadores hombres, por su escasez. 
Una persona del equipo investigador contactó con los cuidadores por teléfono, evitando así que el contacto lo realizará personal del centro de salud, para que los entrevistados no se sintieran condicionados por esta circunstancia. Todos los grupos y entrevistas fueron realizados por la misma entrevistadora, grabados y posteriormente transcritos. Las dinámicas grupales fueron muy intensas y satisfactorias para los participantes, que encontraron en ellas un espacio dónde contar sus vivencias y compartirlas entre «iguales». El nivel de acuerdo fue muy alto. Todos los grupos duraron entre $120 \mathrm{y}$ 180 minutos. Los participantes en los grupos no se conocían entre sí, ni tampoco conocían a la entrevistadora. Las técnicas grupales se realizaron en locales ajenos a los centros de salud. Las entrevistas en profundidad duraron alrededor de 90 minutos. Los entrevistados se sintieron satisfechos y agradecidos por el hecho de que alguien se interesara por su experiencia y sus dificultades en el cuidado del enfermo. Las entrevistas se realizaron en el domicilio del cuidador. En todos los casos la disposición a participar ha sido máxima.

Procedimiento de análisis de la información. La información se ha analizado mediante análisis de contenido apoyado por el software NUDIST- 4. Las variables o categorías de análisis han sido las definidas en el modelo SERVQUAL de calidad percibida (Parasuraman) (tabla 1). El proceso de análisis se ha realizado en las siguientes fases que se muestran en la figura 2 . La codificación de cada una de las transcripciones (la asignación de las unidades de texto a cada categoría de análisis, en cada una de las transcripciones) se ha realizado mediante triangulación (contraste) entre los miembros del equipo investigador para garantizar la fiabilidad de este proceso. De igual forma se han triangulado los resultados obtenidos en cada variable y en cada perfil de cuidadores. El apoyo del sofware Nudist en la categorización y en el análisis permite la triangula- 


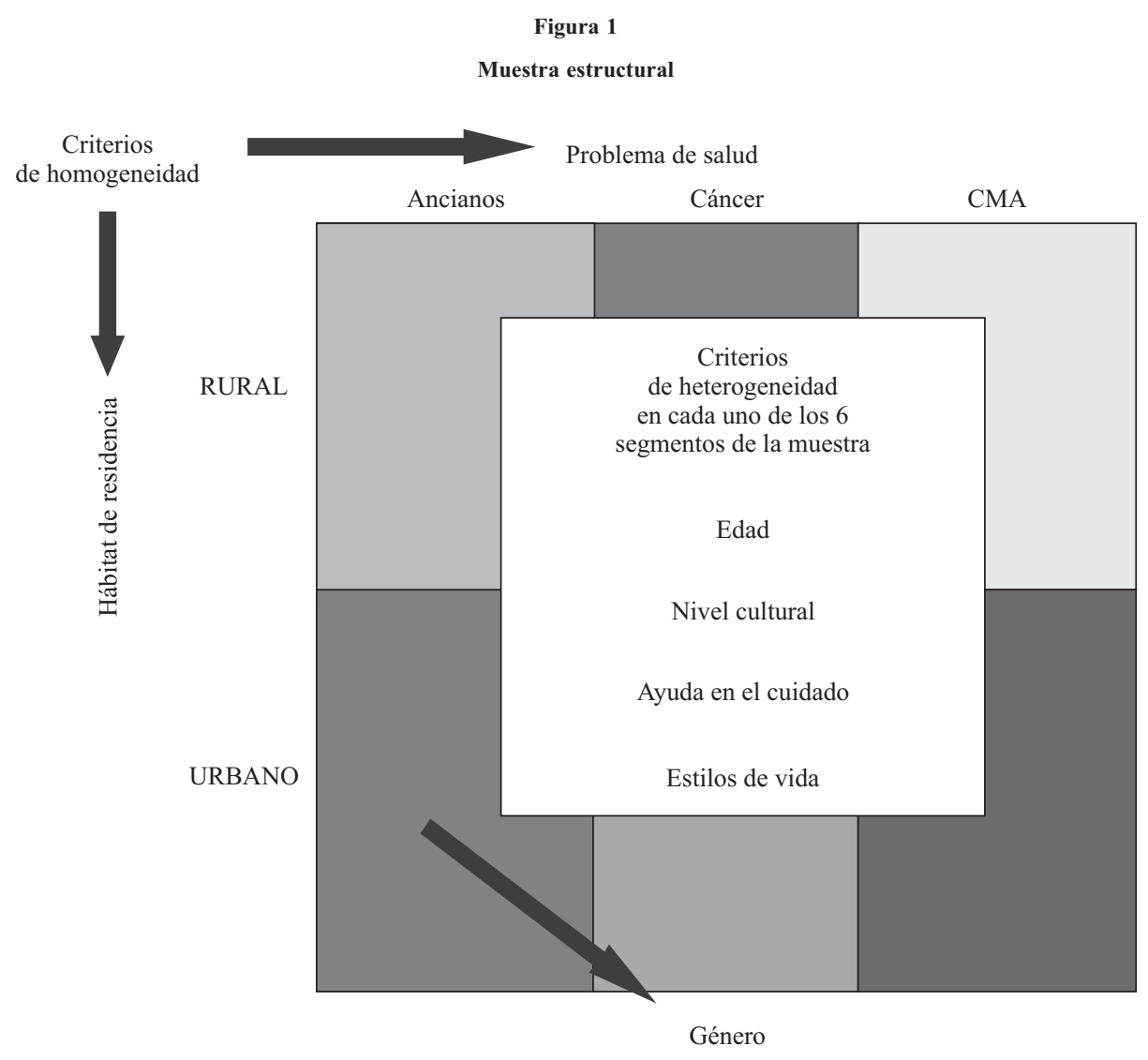

ción de todo el proceso entre el equipo investigador y sistematiza el análisis, proporcionando criterios de rigor y calidad a los resultados, al aumentar la credibilidad, la consistencia y estabilidad de los «datos» y la confirmabilidad de los mismos ${ }^{28}$.

Consideraciones éticas. La participación de los sujetos entrevistados en el estudio se ha realizado previa información de los objetivos del proyecto, de la institución que lo realiza y de la que lo financia y mediante su consentimiento verbal. Se pidió autorización para grabar sus discursos, tanto en los grupos como en las entrevistas. Se les garantizó la confidencialidad de sus opiniones. Se les garantizó que las transcripciones sólo serían escuchadas por los miembros del equipo investigador. En ningún caso aparecen en las transcripciones los nombres reales de los participantes. Se han suprimido de las copias de las transcripciones a disposición de los lectores interesados, todas aquellas referencias que pudieran identificar a los entrevistados.

Limitaciones. Las opiniones de usuarios de la atención domiciliaria, obtenidas a través de metodología cualitativa no podrán ser extrapoladas a la población general, como es característico de este abordaje metodológico. El sesgo de selección de los participantes se ha controlado mediante la estrategia de que sea un miembro del equipo investigador el que realice la selección de los participantes a partir de los listados ela- 


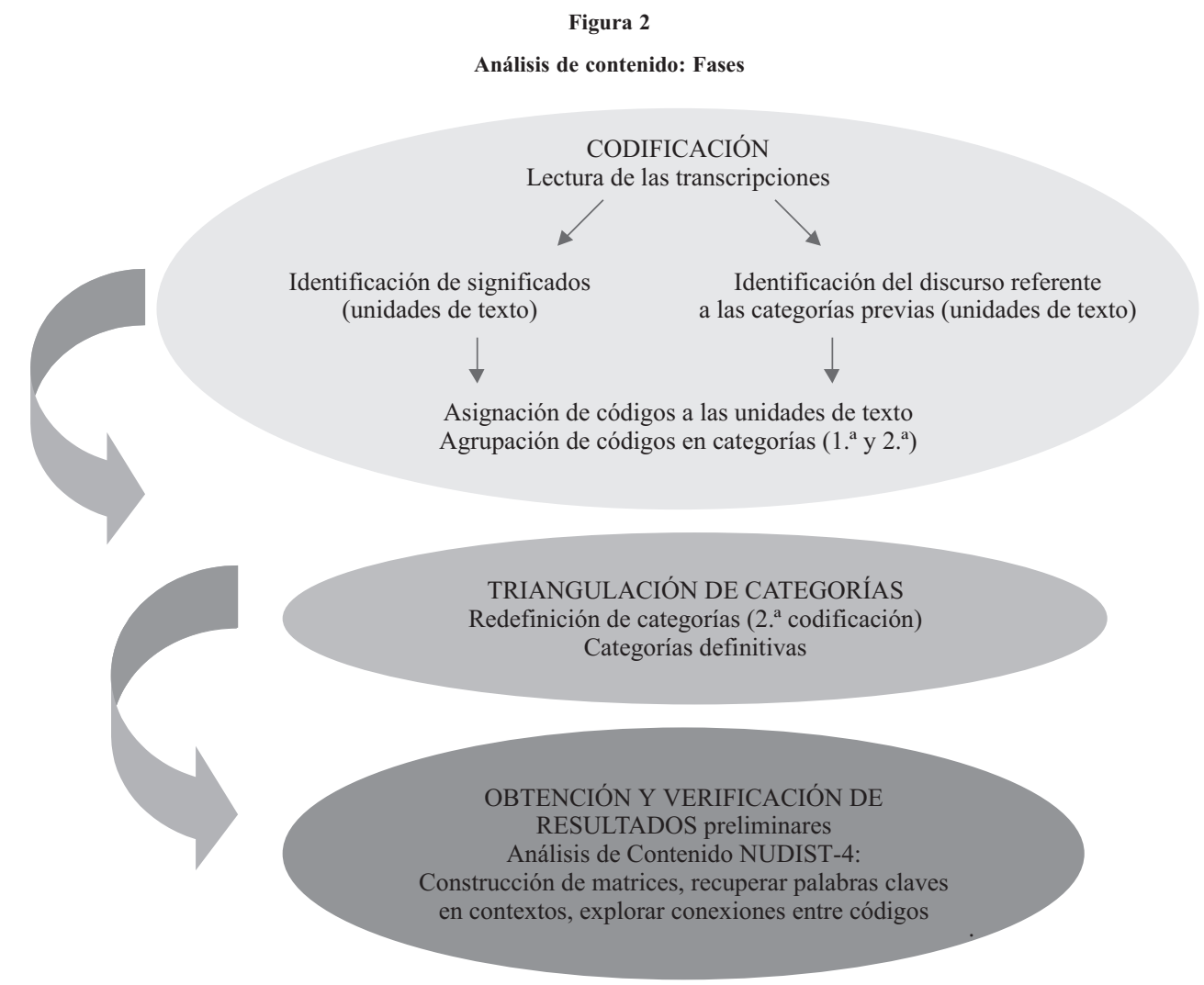

borados por el personal sanitario. No obstante, estos listados podrían no incluir a personas más críticas o conflictivas con la atención domiciliaria que se les proporciona. Además, los listados incluyen, obviamente, a las personas incluidas en programa domiciliario por lo que quedan fuera de esta investigación aquellos sujetos que, necesitando cuidados domiciliarios, no les son proporcionados por los centros de salud (reciben atención domiciliaria de los equipos hospitalarios, de asociaciones de voluntarios, de servicios privados o no reciben ningún tipo de cuidado a domicilio).

\section{RESULTADOS}

(La letra en cursiva corresponde al discurso literal de los entrevistados)
Las dimensiones del SERVQUAL referidas a la $\mathrm{AD}$ sobre las que más hablan los entrevistados en los grupos y entrevistas (línea superior de la figura 3) son la «competencia de los profesionales» seguida de la «capacidad de respuesta», la «accesibilidad y la comunicación». Sin embargo, si analizamos estos resultados según el tipo de paciente al que cuidan, observamos importantes diferencias en el peso que adquieren los diferentes aspectos de la calidad percibida de la $\mathrm{AD}$ que han recibido. La figura 4 muestra las diferencias más significativas. ¿Por qué se establecen estas diferencias en los discursos de las cuidadoras?

1. ¿Qué dicen las cuidadoras de los enfermos de cáncer terminal con respecto a la capacidad de respuesta y la accesibilidad que hacen que estas dimensiones sean las 
Peso relativo de las dimensiones del SERVQUAL en los dicursos de los cuidadores

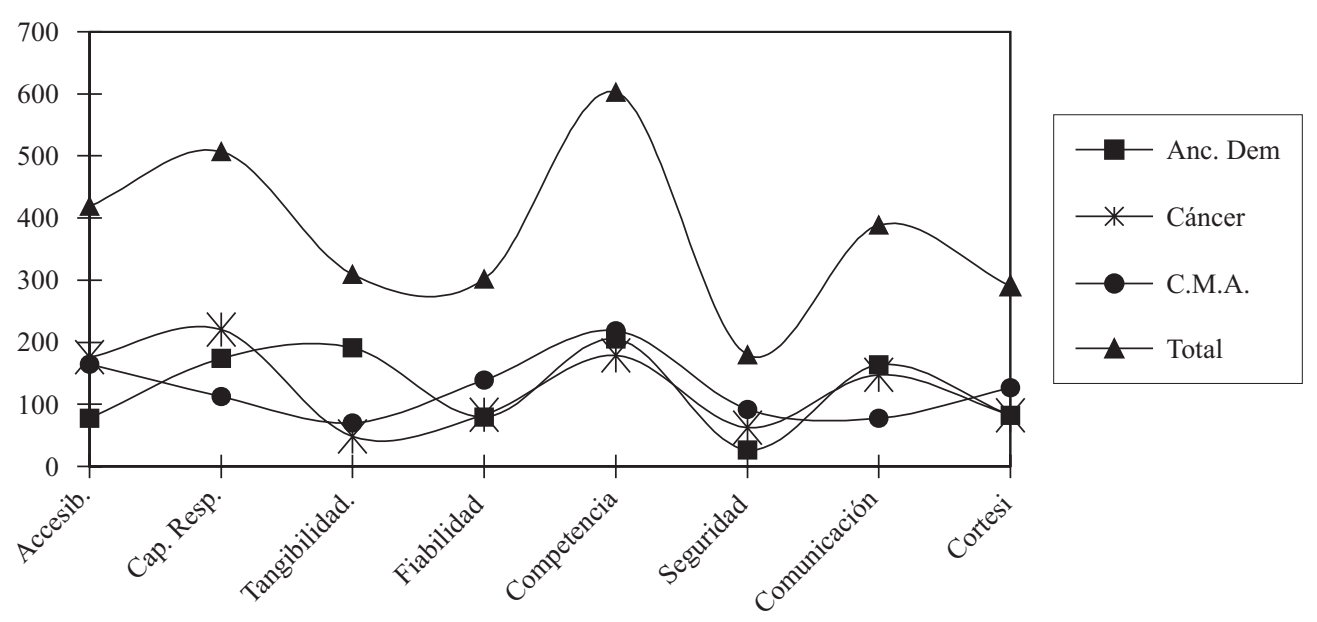

Figura 4

Comparación del peso relativo de las dimensiones del SERVQUAL en los discursos de los cuidadores

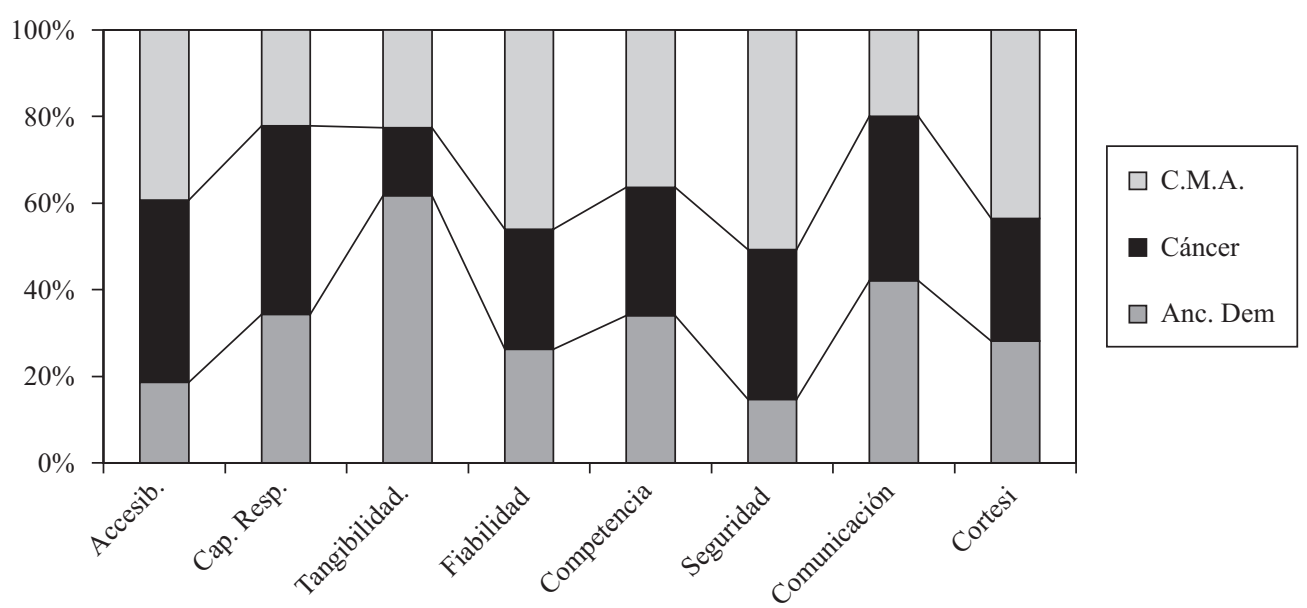


más mencionadas en sus discursos? Estas mujeres opinan que un servicio de $\mathrm{AD}$ debe ser muy accesible y además contar con profesionales capaces de resolver cualquier problema que se le presente al paciente, bien ellos mismos o bien derivándolo al hospital. Aparecen expectativas sobre ello muy bien formadas. Relatan que el problema más frecuente y temido es el dolor. La mayoría de las entrevistadas asegura que los pacientes sufrieron dolor y que éste no se controlaba. Este hecho lo atribuyen a la demora en acudir al domicilio, aunque en muchas ocasiones disculpan al profesional y acusan al sistema. En muchos casos, son las cuidadoras las que administran los analgésicos y opiáceos, utilizando incluso la vía parenteral.

- Es que no puedes esperar, es que se desespera, porque como se ponen nerviosos, es peor. Le temen al dolor.

- Es peor la espera.

- Es peor, cuando ya está desesperado, entonces le pones morfina pura y no se le quita el dolor.

- Tienes que darle primero un relajante, que es con lo que a mí me enseñaron, Tranxilium 50 y un Nolotil, líquido. En la ampolla del Nolotil, se diluye la ampolla del Tranxilium 50 y se hace una pasta muy homogénea, entonces se la pones...

El dolor y el resto de síntomas, la experiencia de algunos episodios críticos en el avance de la enfermedad y el hecho de vivir esperando que suceda la muerte del familiar (que en muchos casos se imagina como un momento trágico y dramático) hace que las cuidadoras necesiten que el equipo de $\mathrm{AD}$ esté accesible y sea capaz de responder con agilidad y rapidez si es necesario.

Sobre la calidad percibida de la AD en estas dimensiones (accesibilidad y capacidad de respuesta) se recogen discursos contrapuestos. Entre los críticos aparecen como elementos negativos: la falta de cobertura de
AD fuera del horario normal del CS, la dificultad de contactar por teléfono con el centro en caso de necesidad, la tardanza en acudir cuando se les llama y el hecho de que los enfermeros no programen las visitas a este tipo de pacientes y acudan al domicilio sólo a demanda.

- Dificil no, dificilísimo, el otro día llamé, pues serían ya, no se, las una y pico que ya, ya están todas las consultas, pues no habia, y comunicaba y comunicaba, y digo, pero, como lo ponen para que comunique, que lo descolgarán.

- Ese es el problema porque mi padre una noche que era que rabiaba de dolor, de no poder estar...

- Tardan muchísimo. Había veces que tenía que estar a cada instante llamando. $Y$ me decian, no, no se preocupe que lo tienen anotado y que ya se pasarán por allí...

- Sin llamarlas no van, ya cuando ven una cosa muy grave, si van. Ya van como una amistad como una cosa de caridad, o de cariño, pero sin llamarlas no van, sin llamarlas no van, ninguna.

2. ¿Por qué la mayoría de los comentarios y referencias que los cuidadores de pacientes intervenidos en CMA realizan en sus discursos giran en torno a la Seguridad y la Fiabilidad de la AD que han recibido?

Para estas personas, el paciente al que cuidan está en una situación inestable y temen que aparezca alguna complicación o que vaya hacia atrás. En la mayoría de los casos se quejan de la premura del alta y hubiesen preferido pasar al menos la primera noche en el hospital.

- ...pensaba que iba a quedar, pero no, no la dejaron. Entonces, pues ya te digo, y entonces también al estar aquí más a las afuera del pueblo pues peor, también porque dice: madre mía que hago yo se pone peor o lo que sea?, qué hacemos?, y mi 
padre tampoco tiene coche ni nada y la verdad.

Por ello, esperan que el equipo de AD tenga los conocimientos, habilidades y recursos necesarios para realizar las curas y aplicar los tratamientos de forma tan segura «como lo harían en el hospital». La comparación y la referencia al hospital es una constante en los discursos de estos cuidadores. Sobre la calidad percibida de AD en el caso de la CMA, la mayoría de los entrevistados realizan una buena valoración de la práctica enfermera. No obstante hay que señalar que, en los casos en los que los cuidadores asumieron la realización de las curas y la aplicación de tratamientos (incluida los parenterales), aunque en muchos casos reconocían que las enfermeras les habían enseñado, sus opiniones eran críticas respecto a ello y describían la experiencia como angustiosa por la inseguridad con la que habían pasado estos días.

- Las inyecciones se las tuve que poner yo, todos los días que, no me acuerdo cuántos, pero todos los días se las puse yo.

- Tienes que curarla. Me fui acostumbrando, porque al principio me costó, y creía que lo hacía mal y que la herida se le podia abrir.

Manifiestan los entrevistados que, para que los enfermeros acudan a la casa, éstos deben valorar la cura como «importante» y explican que debido a la presión asistencial no les queda tiempo para realizar curas «leves» en los domicilios.

- Ellos curan escaras y cosas así gordas.

- Pero dicho por una enfermera, que cosas así leves, que para ellos no es una cura importante, que no vienen a hacerla.

Este hecho genera actitudes distintas entre los entrevistados. Unos entienden que el personal de enfermería debe reservarse para cosas más importantes y, sin embargo, otros consideran que el paciente necesita de cui- dados profesionales, aunque el problema no sea de mucha envergadura. De hecho, muchos de éstos hacen el esfuerzo de desplazarse hasta el centro de salud para que les curen allí y otros, incluso, hasta el hospital de referencia.

3. ¿Qué preocupa o interesa a los cuidadores de ancianos con demencia sobre los recursos materiales que proporcionan las enfermeras y a los que dedican gran parte de sus discursos en los grupos y entrevistas?

Los entrevistados presentan muchas quejas en cuanto a los materiales y recursos que necesitan para el cuidado del familiar. El tiempo que le dedican a hablar sobre esta variable es siempre para referirse a la insuficiencia de los mismos. Según ellos no disponen de la cantidad necesaria de gasas, guantes o antiséptico. También piden colchones antiescaras y más cantidad de pañales.

- Y pañales uno al día. Lo que yo he pasado...

- Yo a mi madre, hay tardes que necesita 6 pañales, entonces hay que costearlo ya porque no me dan más que 4.

- Hay veces que por lo que sea necesitas 2 pañales $y$, entonces ya te está faltando.

- Este es el problema que hay.

Aunque entienden que la solución a este problema no está en manos de la enfermera, en los casos en los que ésta ha intervenido aconsejando, mediando o facilitando la obtención de recursos, la valoración de su competencia profesional es muy alta.

\section{DISCUSIÓN}

Dado que la calidad percibida por el usuario y su satisfacción están basadas en preferencias subjetivas o expectativas que no necesariamente se relacionan con la calidad objetiva de la asistencia sanitaria, consideramos 
el abordaje cualitativo como el más idóneo para estudiar estos aspectos. Además, esta metodología permite la participación de los usuarios en la evaluación y mejora de los servicios $^{27}$. Esto nos parece especialmente importante en el caso de la $\mathrm{AD}$, sometida a un intenso debate sobre su futuro ${ }^{32-35}$.

Los criterios utilizados para la definición de los perfiles más característicos de los cuidadores (tabla 2) coinciden con los señalados en los estudios realizados en Andalucía y España sobre cuidadores informa$\operatorname{les}^{23-25,36,37}$. Por ello creemos que los resultados obtenidos pueden ser transferidos o aplicarse a otros sujetos o contextos similares a los del presente estudio. La transferibilidad de los resultados es uno de los criterios de calidad en la investigación cualitativa (criterios de confiabilidad de GUBA) ${ }^{28}$.

Hay que señalar que en los grupos y entrevistas había personas con diferentes actitudes y valoraciones (unas más positivas y otras más negativas) hacia la AD que habían recibido, lo que muestra que no se habían seleccionado sólo a los más satisfechos.

Sobre los resultados obtenidos señalar en primer lugar que la principal necesidad de los cuidadores de pacientes oncológicos terminales es que el equipo de AD sea capaz de aliviar el dolor del paciente. Sin embargo, en la experiencia de algunos de los entrevistados esto no ha sucedido. El control del dolor es uno de los principales indicadores de calidad asistencial utilizados por la OMS. Estudios recientes revelan que un importante número de profesionales de atención primaria no utilizan la morfina o no lo hacen correctamente $^{38,39}$. Y no hay que olvidar que el $90 \%$ de este tipo de pacientes experimentan dolor, siendo en muchos casos la principal razón del deterioro de la calidad de vida en sus últimos días y un motivo importante de sus visitas a urgencias e incluso de que acudan a morir al hospital ${ }^{40,41}$.

Sobre la accesibilidad de los profesionales al domicilio del paciente queremos men- cionar que en un estudio realizado en Andalucía durante el 2000 , el $54 \%$ de los pacientes oncológicos terminales de un distrito sanitario no recibieron nunca $\mathrm{AD}^{42}$. $\mathrm{La}$ queja de los cuidadores sobre la falta de programación de las visitas podría significar que el intervalo entre ellas lo consideran muy largo. Si esto es así, habría que revisar los criterios de la programación y ajustarlos a las necesidades y características de cada familia, teniendo presente que, el acudir a la casa sin que tener que llamarlos es un indicador de calidad para el paciente y acudir sólo a demanda, todo lo contrario. Sin embargo, no se puede obviar que la práctica de enfermería en atención primaria, hoy por hoy, y en la mayoría de los centros, está centrada en la consulta de enfermería, priorizando esta actividad frente al resto. Así se revela en un estudio realizado en Andalu cía ${ }^{43}$ durante el 2000: el 40,4\% de los enfermeros dedicaba más de 3 horas diarias a la consulta de enfermería (de ellos el $27 \%$ dedicaba más de 4) y el 39\% entre 2 y 3 horas cada día. La media horas dedicada a la $\mathrm{AD}$ era de 4,9 semanales ( $\mathrm{S}=3,3$; NC: $95 \%$ )

Sobre los discursos de los cuidadores de pacientes intervenidos en el modelo de CMA queremos destacar la necesidad manifiesta de que el equipo de $\mathrm{AD}$ les proporcione la confianza y seguridad suficiente como para minimizar el desamparo que han experimentado con el alta precoz. En los estudios publicados sobre la satisfacción de los pacientes y sus cuidadores con el alta precoz no aparece consenso al respecto ${ }^{44-46}$. Lo que sí parece que mejora la aceptabilidad de estos programas es la garantía de continuidad de cuidados al alta, bien desde el propio hospital o desde el $\mathrm{CS}^{47}$. En todo caso se hace necesario que los profesionales de ambos niveles trabajen de forma coordinada, para evitar las contradicciones o las prácticas diferentes que tanto desconciertan al paciente y que son responsables de la inseguridad de éste. La estrategia institucional de la Gestión por Procesos Asistenciales que se está desarrollando en Andalucía puede ser una propuesta eficaz ${ }^{48}$. Junto a esto, los re- 
sultados ponen de manifiesto la necesidad de mejorar la información a los pacientes y familiares sobre las características de los programas de cirugía de alta precoz y de garantizar que el consentimiento del paciente se produce una vez que éste entiende y acepta lo que se le propone. Otros estudios también lo señalan ${ }^{47}$. Y además de todo lo anterior, también parece necesario contemplar la posibilidad de que, en aquellos casos en los que el equipo sanitario no consigue tranquilizar al paciente para que se vaya a casa seguro, pueda permanecer la primera noche en el hospital.

De los discursos de los cuidadores de ancianos, señalar la influencia que tiene en la calidad percibida el que los profesionales muestren interés por los problemas del paciente y del cuidador. Muestra de ello es que, aunque saben que la mayoría de las veces la enfermera no puede proporcionarles los recursos materiales que necesitan, valoran sus gestiones para facilitárselos. En este sentido, parece necesario potenciar la dimensión relacional y de comunicación de la práctica sanitaria, como mecanismo eficaz para mejorar la calidad percibida por los pacientes de los cuidados que reciben. En España, los cuidados de salud de los ancianos dependientes son asumidos casi en su totalidad por sus familias (el cuidado formal representa sólo entre el 2 ó 3,5\% de la ayuda que necesitan ${ }^{25,49}$. El cuidado se considera un deber y una obligación. Esto explica que los cuidadores entrevistados tengan pocas expectativas sobre las ayudas socio-sanitarias para la atención al familiar, que se hace más evidente en al caso de las mujeres por la relación género-rol de cuidadora. Por último, comentar que las demoras de los profesionales en acudir al domicilio que relatan los entrevistados y que genera una falta de capacidad de respuesta ante situaciones urgentes que requieren de atención inmediata, debería hacernos reflexionar sobre los modelos organizativos de los centros que siguen priorizando el trabajo en el CS, y más concretamente, sobre el papel de las enfermeras. Incluso podríamos debatir sobre la capacidad del modelo de CS tradicional para responder de manera satisfactoria a las necesidades y problemas de estos pacientes. ¿Deberíamos pensar en la posibilidad de potenciar los Equipos de Soporte? ¿Tendría sentido la existencia de enfermeras domiciliarias dentro de los equipos de atención primaria?

Concluyendo, la atención domiciliaria es un servicio necesario para las cuidadoras informales de pacientes terminales e inmovilizados que facilita el cuidado en el hogar y garantiza una atención de calidad. Sin embargo desean mejoras en la accesibilidad y en la capacidad de respuesta. La actual oferta se considera insuficiente por la escasa cobertura y la distancia temporal entre una visita y otra. Además, cuando se presenta una situación urgente, la capacidad de respuesta del equipo de atención domiciliaria no se considera satisfactoria. La mejora de las competencias de los enfermeros domiciliarios también se percibe como una expectativa de los usuarios. Los cuidadores desean que las enfermeras domiciliarias tengan los conocimientos necesarios como para resolver los problemas del paciente en casa con la misma garantía y eficacia que en el hospital. Se detecta un problema importante respecto a la capacidad de los profesionales para controlar el dolor de los pacientes terminales.

Las expectativas de cuidadores y pacientes varían en función del problema de salud. Por lo tanto, las características de la atención domiciliaria tendrán que variar también en función de ello. Se hace necesario un modelo flexible que sea capaz de adaptarse a las necesidades de los diferentes tipos de pacientes y las circunstancias también distintas de sus cuidadores familiares.

\section{BIBLIOGRAFÍA}

1. SESPAS. Informe SESPAS 2000: La salud pública ante los desafíos de un nuevo siglo. Granada: EASP; 2000. 
2. Coleman B. European models of long-term care in home community. Int J Health Serv 1995; 25 : 458-74.

3. García Barbero M. From hospitals to home health care: an alternative requiring careful thinking. Copenhague: WHO; 1997.

4. Contel JC, González M. Reflexión sobre la implementación de programas de atención domiciliaria. Enfermería Clínica 5; 5: 205-211.

5. Contel Segura JC. La atención a domicilio como modelo de atención compartida. Aten Primaria 2000; 25: 22-7.

6. Parasuraman A, Zeithaml V y Berry L. A conceptual Model of Service Quality and Its Implications for Future Research. J Mark 1985; 41-50.

7. Parasuraman A y Berry L. Calidad total en la gestión de servicios. Madrid: Diaz de Santos; 1993.

8. Carman JM. Consumer perceptions of services quality: an assessment of the SERVQUAL dimensions. Journal of retailing 1990; 66: 33.

9. Asubonteng P. McCleary JKSJ. SERVQUAL revisited: a critical review of service quality. The $\mathrm{J}$ Prof Serv Mark 1996; 10: 62-81

10. Thompson A, Suñol R. Las expectativas como factores en la satisfacción de los pacientes: conceptos, teorías y pruebas. Revista de calidad asistencial 1996; 74-86.

11. Mira JJ, Buil JA, Rodríguez-Marin J, Aranaz J. Calidad percibida del cuidado hospitalario. Gac Sanit 1997; 11: 176-89.

12. Prieto Rodríguez MA, March Cerdá JC, López Fernández L. A. Calidad percibida por usuarios de centros de salud y de aseguradoras privadas. Aten Primaria 1999; 24: 259-266.

13. Mira JJ, Aranaz J. La satisfacción del paciente como una medida del resultado de la atención sanitaria. Med Clin (Barc) 2000; 114 (supl 3): 26-33.

14. Gea MT, Hernán M, Jiménez.Martín J, Cabrera A. Opinión de los usuarios sobre la calidad del servicio de urgencias del centro médico-quirúrgico del hospital Virgen de las Nieves. Rev Calidad Asistencial 2001; 16: 37-44.

15. Mira JJ, Galdón M, Ignacio García E, Velasco MV, García S, Vitaller J et al. ¿Qué hace que los pacientes estén satisfechos? Análisis de la opinión de pacientes y profesionales mediante la técnica Delphi. Rev Calidad Asistencial 1999; 14: $165-78$
16. Mira JJ, Rodríguez-Marin J, Peset R, Ybarra J, Pérez-Jover V, Palazón I, et al. Rev Calidad Asistencial 2002; 17 (5): 273-83.

17. Shortell SM, Fache PD. La necesidad urgente de métodos cualitativos en la investigación de servicios sanitarios. Health Serv Res 1999; 34 (Part II) (5): 1083-9.

18. March Cerdá JC, Prieto Rodríguez MA, Hernán García M, et al. Técnicas cualitativas para la investigación en salud pública y gestión de servicios de salud: algo más que otro tipo de técnicas. Gac Sanit 1999; 13: 312-319.

19. Pope C, Mays N. Reaching the parts other methods cannot reach: an introduction to qualitative methods in health and health services research. BMJ 1995; 311: 42-5.

20. Fernández de Sanmamed MJ. Métodos y técnicas cualitativas en la investigación en atención primaria. Aten Primaria 1999; 24: 453-4.

21. Sofaer S. Métodos cualitativos: ¿qué son y por qué usarlos? Health Serv Res 1999; 34 (Part II) (5): 1101-18.

22. Hurley RE. La investigación cualitativa y el profundo entendimiento de lo obvio. Health Serv Res 1999; 34 (Part II) (5): 1119-36.

23. Bazo T. Los cuidados familiares de salud a las personas ancianas y las políticas familiares. Revista Española de Investigaciones Sociológicas 1995; (Monografía): 1-22.

24. Duran MA. El cuidado de la salud. En: De puertas adentro. Madrid: Instituto de la Mujer; 1988.

25. García Calvente MM., Mateo Rodríguez IGCP Cuidados y cuidadores en el Sistema Informal de Salud: Investigación cuantitativa. Granada: EASP; 1999.

26. Mays N, Pope C. Rigour and qualitative research. BMJ 1995; 311: 109-12.

27. Iñiguez Rueda L. Investigación y evaluación cualitativa: bases teóricas y conceptuales. Aten Primaria 1999; 23: 496-502.

28. Pla M. El rigor en la investigación cualitativa. Aten Primaria 1999; 24: 295-300.

29. Ruiz Olabuénaga JI. El diseño cualitativo. En: Ruiz Olabuénaga JI ed. Metodología de la investigación cualitativa. Bilbao: Universidad de Deusto; 1996. p. 64.

Rev Esp Salud Pública 2002, Vol. 76, N. ${ }^{\circ} 5$ 
30. Prieto Rodríguez MA, March Cerdá JC. Paso a paso en el diseño de un estudio mediante grupos focales. Aten Primaria 2002. 29: 366-73.

31. Ortí A. El grupo de discusión en su práctica: Memoria social, intertextualidad y acción comunicativa. Revista Internacional de Sociología 1996; 13.

32. Benítez MA. ¿Por qué la atención domiciliaria a debate? Aten Primaria 1999; 24 (supl 2): 163.

33. Gené J, Contel JC. Propuestas para desarrollar la atención domiciliaria. Aten Primaria 1999; 23: $18-20$.

34. Prieto MA, March JC, Pascual N. Propuestas para una atención domiciliaria realista en atención primaria. Aten Primaria 1999; 24: 439.

35. Contel JC. Modelos de Atención a domicilio en el entorno europeo. Aten Primaria 1999; 24 (supl 2): $163-5$.

36. Mateo Rodríguez I, Millán Carrasco A, Garcia Calvente MM, Gutierrez Cuadra P, Gonzalo Jiménez E, López Fernández LA. Cuidadores familiares de personas con enfermedad neurodegenerativa: perfil, aportaciones e impacto de cuidar. Aten Primaria 2000; 26: 139-44.

37. Roca Roger M, Ubeda Bonet I, Fuentelsaz Gallego $\mathrm{C}$ et al. Impacto del hecho de cuidar en la salud de los cuidadores familiares. Aten Primaria 2000; 26(4): 217-23.

38. Benítez del Rosario MA, Salinas Martín A, Asensio Fraile A, Armas J. Cuidados paliativos en atención primaria: opinión de los profesionales. Aten Primaria 1999; 23: 187-191.

39. Busquet Durán X, Porta Serra M, Jariod M, Busquet Mas C, Sánchez Blanco AM, Duocastella Bastardes P. La atención domiciliaria al enfermo de cáncer terminal: valoración y propuestas de médicos y enfermeras. Aten Primaria 1994; 13: 291-297.

40. Seamark DA, Thorne CP, Lawrence C, Pereira Gray DJ. Appropiate place of death for cancer pa- tients: views of general practitioners and hospital doctors. Br J Gen Prac 1995; 45: 359-363.

41. De Conno F, Caraceni A, Groff L, Brunelli C, Donati I, Tamburini M, et al. Effect of Home Care on the Place of Death of Advanced Cancer Patients. Eur J Cancer 1996; 32: 1142-1147.

42. Prieto MA. Satisfacción de usuarios con la atención domiciliaria a pacientes oncológicos (FIS/99/0047-03). Memoria científica 2000. Granada: Escuela Andaluza de Salud Pública; 2001.

43. Prieto MA, Heierle C, Frías A, Calzada ML, Alcaide A, Vázquez T, Sandino L. Práctica enfermera en atención domiciliaria: opinión de profesionales. (FIS/98/0653). Granada: Escuela Andaluza de Salud Pública; 2001.

44. Shepperd S, Ilife S. Effectiveness of hospital at home compared to in-patient hospital care (Cochrane review). In The Cochrane Library, Issue 3, Oxford: Update Software; 1998.

45. Richards SH, Coast J, Gunnell DJ, Peters TJ, Pounsford J, Darlow MA. Randomized controlled trial comparing effectiveness and acceptability of an early discharge, hospital al home scheme with acute hospital care. BMJ 1998; 316 : 1796-1801.

46. Sola ME, Bernuz A, Lázaro JJ, Bosch X, Cáncer L, Serra M. Cirugía ambulatoria pediátrica: una nueva oferta sanitaria rentable ¿y además satisfactoria? Rev Calidad Asistencial 1998; 13: 6-12.

47. Caplan GA, Brown A, Crowe PJ, Yap S, Noble S. Re-engineering the elective surgical service of a tertiary hospital: a historical controlled trial. Med J Austr 1998; 168: 247-51.

48. Consejería de Salud. Junta de Andalucía. Plan de Calidad. Nuevas estrategias para la sanidad andaluza. Sevilla: Consejería de Salud; 2000.

49. Valderrama E, Ruiz D, Adame JF, García C, García PA, Pérez del Molino J. El cuidador principal del anciano dependiente. Rev Gerontol 1997; 7 : 229-233. 